\title{
PAPR Reduction of OFDM Systems using 2D Inverse Discrete Fourier Transform
}

\author{
Waleed Khaleel Hassoon \\ Department of Computer \\ Engineering Technology, Al- \\ Salam University College, \\ Baghdad, Iraq
}

\author{
Ali Abdul Kadhum Ruhaima \\ AL-Nisour University College, \\ Baghdad- Iraq
}

\author{
Mokhalad Khaleel Alghrairi \\ Al-Mustansiriya University \\ Iraq
}

\begin{abstract}
A tow-dimensional (2D) orthogonal frequency division multiplexing (OFDM) system with peak-to-average power ratio (PAPR), a multicarrier system, is an important part of wireless communications engineering. Since these signals divide the bandwidth into other sub channels n, so the OFDM signals accomplish top data rates. Although, High peak-toaverage power ratio (PAPR) of the transmit signal is a major problem of multicarrier transmission such as orthogonal frequency division multiplexing (OFDM). To make fewer the PAPR values, There are many of techniques have been introduced, including selected mapping and amplitude clipping. The method that produces the least bit-error rate (BER) performance, its method is Amplitude clipping and filtering which is the simplest method. And from the other side, SLM although it presents a computational complexity, it is considered the most suitable and distortion less. In this research, a new technique is proposed based on twodimensional discrete Fourier transform (2D-DFT). In this paper we present a new method that reduces the computational complexities and distortion less compared to the SLM method. The M-PSK mapping types show from theoretical analysis that the PAPR can be reduced to something like $0.0 \mathrm{~dB}$, as proven mathematically and by computer simulations.
\end{abstract}

\section{Keywords}

2D-DFT; CCDF; Orthogonal frequency division multiplexing modulation; PAPR reduction, SLM technique, complexity.

\section{INTRODUCTION}

Orthogonal Frequency Division Multiplexing (OFDM) is a digital multi-carrier modulation scheme that extends the concept of single subcarrier modulation by using multiple subcarriers within the same single channel [1]. It is an important technology because many developing communications standards require the high throughput and multi-path advantages that are possible, Using a large number of parallel narrow band sub carriers instead of a single wide band carrier to transport information, Some of the benefits of using OFDM signals it's very easy and efficient in dealing with multi-path and Robust again narrow-band interference. And from the disadvantages, it can be said that sensitive to frequency offset and phase noise and the peak-to-average problem reduces the power efficiency of RF amplifier at the transmitter. The main concept in OFDM is orthogonally of the sub-carriers. Since the carriers are all sine/cosine wave, we know that area under one period of a sine or a cosine wave is zero. Several common commercial protocols, such as digital video broadcast (DVB), asymmetric digital subscriber line (ADSL), and wireless Ethernet (WiFI) implement OFDM.

OFDM communications systems are able to more effectively utilize the frequency spectrum through overlapping subcarriers. These sub-carriers are able to partially overlap without interfering with adjacent sub-carriers because the maximum power of each sub-carrier corresponds directly with the minimum power of each adjacent channel.

Peak-to-average power ratio (PAPR) is proportional to the number of subcarriers used for OFDM systems. An OFDM system with large number of subcarriers will thus have a very large PAPR when the subcarriers add up coherently. Large PAPR of a system makes the implementation of digital-toanalog converter (DAC) and analog-to-digital converter (ADC) extremely difficult. The design of RF amplifier also becomes increasingly difficult as the PAPR increases. One of the benefits of using OFDM signals is a high-output at the high power amplifier (HPA). Always HPA is a linear but when the signal reaches the saturation area, the HPA is called

A non-linear device. Inter-modulation distortion occurs when the high peaks of the OFDM signal, this causes the HPA in the non-linear region.

The technology is classified into the distortion and distortion less subclasses.

To manage high PAPRs, The first type includes the methods high bit-error rate (BER) performance, such as filtering (ACF) [2], the signals in the frequency domain deals with the SLM method [3], where in $U$ copies of the signal correspond to $U$ phase-rotation vectors, Each copy is multiplied by its corresponding phase-rotation vector. The value of the PAPR shall be as low as possible in this combination which is sent to the transmitter, with the phase-rotation vector index as side information. $\log 2$ represents the number of bits in the side information.

So high computational complexity leads to the largest $\mathrm{U}$ because of the use of $U$ blocks of inverse discrete Fourier transform (IDFT) operations. Alternatively, PTS [4] is also distortion less and can reduce the PAPR in a manner similar to SLM [5], but with more computational complexity as well as similar information as the SLM. Meanwhile, the coding techniques [6] are distortion less, lead to a marked decrease in the PAPR values. However, a drop in the data bit rate is also observed. Other mechanisms can be used to decrease the PAPR but not within the limits of this research.

In this paper, the method was used to lead to non-low bit rate as well as BER deterioration; this method is called the discrete Fourier transform (DFT). This method can be used to decrease 
the PAPR at less complexities compared with SLM techniques and without requiring side information. To reduce the PAPR can be considered a distortion less technique be used the IDFT/DFT pair. However, the application of this way is limited by its high cost: it can only be executed with Mlevel based OFDM signals. This method is used with OFDMbased M-QAM mapping signals (which results in lesser PAPR decrease) [7]. In the next sections, we will show why the M-QAM signals did not follow the same performance of the M-PSK signals. The rest of this research is organized as follows: the basic principles of the OFDM system is presented in section 2 along with the PAPR problem; the proposed system and its PAPR analysis are discussed in section 3; the discussion of the results is in section 4 , and our conclusions for the proposed scheme is given in the last section.

\section{OFDM AND PAPR DESCRIPTION}

By using a single carrier a stream of binary data can be transmitted over a wireless channel. This signifies that each bit or symbol (e.g., QPSK) can in sequence are running the entire bandwidth of a channel. The OFDM modulation system is a famous type of modulation where the sub-carrier waves are orthogonal. By using the IDFT process, the OFDM signals are simply obtained, as illustrated in Figure 1 (below).

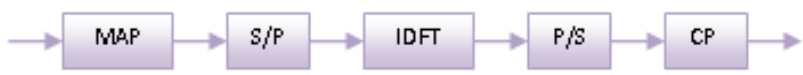

\section{Fig 1: Basic OFDM transmitter}

In Figure one, The M-PSK represents the first box in Fig, the serial-to-parallel converter represents the second square in the figure, the third is the inverse discrete fast Fourier transformation operation, the parallel-to-serial converter represents the fourth square in Fig, the cyclic prefix insertion block it represents the last box. Figure 1 can also be represented mathematically as follows IDFT expression:

$$
x(k)=\frac{1}{\sqrt{N}} \sum_{n=0}^{N-1} X(n) \exp \left(j 2 \pi \frac{k n}{N}\right), n, k=0,1 \ldots N-1
$$

Where, the input M-PSK signal samples are given by X (n) and $\mathrm{x}(\mathrm{k})$ is the time-domain output signal. Figure one Illustrates the OFDM signal is in the baseband and no radio frequency carrier. Therefore, always be a discrete signal that must be approximated from its analog version. By sampling X (n) by a factor, this can bring approximation. Number four is the appropriate value and occasion for L, as stated by [8]. The signal $\mathrm{x}(\mathrm{k})$ is added constructively in the same phase, leading to high peaks compared to the average power. Hence, the PAPR as follows shows that:

$P A P R=\frac{\max |x(k)|^{2}}{P_{a v g}}$

Of the PAPR has widely used the supplementary cumulative distribution function to find out the performance of the system with regard tothe PAPR,

$$
C C D F(P A P R)=\operatorname{Pr}\left(P A P R>P A P R_{o}\right)
$$

The clipping level here represents PAPRo. In this work, the M-PSK mapping-based OFDM signal was dealt with accurately to select a specific and appropriate property in this mapping type. On the premise that the binary PSK is $\{-1$,
$+1\}$, then the M-PSK signal It can be said that it represents the following:

$$
X_{M P S K}=\left\{1, \exp \left(j \frac{2 \pi}{M}\right), \ldots \exp \left(j \frac{2 \pi(M-1)}{M}\right)\right\}
$$

So the mapping order is M. Hence, In Equation 4 it was revealed that $\mathrm{N}$ is the same

the envelope power of all M-PSK signals. Consequently, the constant difference is

$$
\begin{aligned}
\sigma_{M P S K}^{2} & =\frac{1}{N} \sum_{n=0}^{N-1}|X(n)|^{2} \\
& =\frac{N}{N}=1
\end{aligned}
$$

The required property in this work is illustrated in Equation 5. The next section will then discuss the application of M-PSKbased OFDM signals in the proposed technique.

\section{THE PROPOSED METHOD}

In the figure number one has shown the structure OFDM system Traditional. A two-dimensional IDFT (2D-IDFT) based on this proposed system as shown below are shown in Fig. 2 with M-PSK mapping.

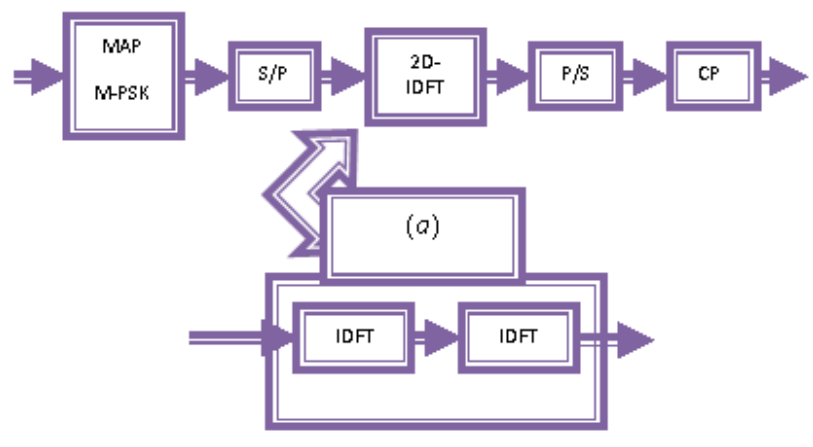

(b)

Fig 2: The suggested OFDM system

a) The complete block diagram implying M-PSK and 2DIDFT,

\section{b) The implementation of the 2D-IDFT is a cascade of 2} IDFT blocks.

The use of (2D-IED) has been done in comparison to IDFT in this new system as shown, the 2D-IDFT block which is a series of two IDFT blocks. It can produce an exact 2D-IDFT but for the similar input information path as in Figure 2a. In Figure $2 b$, the cascade must be done in sequence. This process can be expressed as

Let: F-1 $\{f\}$ is the IDFT of vector $f$, then

Take up F-1 $\{\mathrm{F}-1\{\mathrm{f}\}\}=2 \mathrm{D}-\mathrm{IDFT}$

Usually, the 2D-IDFT can be said :

$$
f(r, n)=\frac{1}{R N} \sum_{u=0}^{R-1} \sum_{v=0}^{N-1} F(u, v) \exp \left(j 2 \pi\left(\frac{r u}{R}+\frac{n v}{N}\right)\right)_{(6)}
$$


where $u=0,1 \ldots r-1$ and $v=0,1 \ldots N-1$. Thus, Equation 6 , can be rewritten as

$$
f(r, n)=\frac{1}{R} \sum_{u=0}^{R-1}\left\{\frac{1}{N} \sum_{v=0}^{N-1} F(u, v) \exp \left(j 2 \pi \frac{n v}{N}\right)\right\} \exp \left(j 2 \pi \frac{u r}{R}\right)_{(7)}
$$

The associated term in Equality 7 is one-dimensional IDFT, which be able to be straight calculated using the fast IDFT style (IFFT) of the rth row. Later, every link is replaced by its IDFT, and the 1D-IDFT of all column can be calculated via the IDFT or the typical IFFT.

In this type of situation, $\mathrm{R}=\mathrm{N}$. That is, the 2D-IDFT are represented in Figure 2b nonetheless only used for the onedimensional input information path $\mathrm{X}(\mathrm{n})$. Agreement $\mathrm{S}(\mathrm{r})$ be the output of the first IDFT block. Then and there,

$$
S(r)=\frac{1}{N} \sum_{n=0}^{N-1} X(n) \exp \left(j 2 \pi \frac{r n}{N}\right)
$$

In addition

$$
x(k)=\frac{1}{N} \sum_{m=0}^{N-1} S(r) \exp \left(j 2 \pi \frac{r k}{N}\right)
$$

Otherwise (as one expression similar to Equation 7),

$$
x(k)=\frac{1}{N} \sum_{r=0}^{N-1}\left[\frac{1}{N} \sum_{n=0}^{N-1} X(n) \exp \left(j 2 \pi \frac{r n}{N}\right)\right] \exp \left(j 2 \pi \frac{r k}{N}\right)
$$

Anywhere $\mathrm{m}, \mathrm{n}, \mathrm{k}=0,1 \ldots \mathrm{N}-1$. Therefore, Equation 8 is the first IDFT block although Equation 9 is the second block; namely, Equation 9a special delivery the processes in Figure $2 b$.

At this moment, an interesting way that calculates the PAPR significance previous to feeding the information vector, $\mathrm{X}(\mathrm{n})$, to the IDFT block is obtainable [9]. Let the autocorrelation method it is expressed in the following equations:

$$
C(l)=\sum_{n=0}^{N-1-l} X(n+l) X^{*}(n), \quad l=0,1 \ldots N-1
$$

the aperiodic autocorrelation function have the amount of the total values it is expressed in the following equations:

$$
\Phi=\sum_{m=1}^{N-1}|C(m)|
$$

Consequently, PAPR can be represented as follows:

$$
P A P R \leq 1+\frac{2}{N} \Phi
$$

Therefore, if $\Phi$ is decreases, the PAPR will be decreases. This approximation practise was correspondingly used in literature [10]. In this paper, M-QAM signals were not considered because Equation 12 cannot be used for the M-QAM constellations, as stated in $[11,12,13,14,15$, and 16] and as proven in $[17,18,19]$. The upper limits for M-QAM are given in the literatures $[20,21]$. Thus, only M-PSK is considered here because of the aforementioned reason as well as by the special case stated in Equations 4 and 5. Therefore, in this work proved that the M-PSK signals can produce a just about constant PAPR and that the M-PSK signals up to about 0.0 $\mathrm{dB}$ using the 2D-IDFT method.

By way of up to that time mentioned, the M-PSK signal is expected to produce up to about $0.0 \mathrm{~dB}$ next the second IDFT process. To show this assumption, Equation 5 (rewritten here for easy reference) is first examined:

$$
\sigma_{M P S K}^{2}=\frac{1}{N} \sum_{n=0}^{N-1}|X(n)|^{2}=\frac{N}{N}=1
$$

Once the head IDFT, Parseval's relation states that the difference will be divide on the vector length, $\mathrm{N}$, by means of:

$$
\begin{aligned}
& \sigma_{1}^{2}=\sum_{m=0}^{N-1}|S(m)|^{2}=\frac{1}{N} \sum_{n=0}^{N-1}|X(n)|^{2} \\
& \therefore \sigma_{1}^{2}=\frac{1}{N} \sum_{n=0}^{N-1}|X(n)|^{2}
\end{aligned}
$$

However,

$$
\sigma_{M P S K}^{2}=\frac{1}{N} \sum_{n=0}^{N-1}|X(n)|^{2}=1
$$

At that time

$$
\sigma_{1}^{2}=\frac{1}{N} \sigma_{M P S K}^{2}
$$

As of this equation,

$$
\sigma_{1}^{2}=\frac{1}{N}
$$

In the exits of the second IDFT and by once more via Parseval's theorem, The following were obtained:

$$
\begin{aligned}
\sigma_{2}^{2} & =\sum_{k=0}^{N-1}|x(k)|^{2} \\
& =\frac{1}{N} \sum_{m=0}^{N-1}|S(m)|^{2} \\
& =\frac{1}{N} \sigma_{1}^{2}
\end{aligned}
$$

Therefore,

$$
\sigma_{2}^{2}=\frac{1}{N^{2}}
$$

The $\Phi$ for the M-PSK signals in this equation prove that the significantly reduced. Nevertheless, the conventional inverse discrete Fourier transform it is possible to say that the expression is capable of the equation:

$$
x(k)=\frac{1}{\sqrt{N}} \sum_{n=0}^{N-1} X(n) \exp \left(j 2 \pi \frac{n k}{N}\right)
$$

The factor 1/N1/2 was not used in our proposed work. Instead, we used 1/N to perform the transformation in Equation 16, as follows:

$$
x(k)=\frac{1}{N} \sum_{n=0}^{N-1} X(n) \exp \left(j 2 \pi \frac{n k}{N}\right)
$$


This equation further verifies the feasibility of our suggested technique. Later the M-PSK based OFDM signals that the average power was proven to continuously equal $1 / \mathrm{N} 2$, the maximum power for this system had to be determined. The maximum peak power cannot be prophesied at the output of the first IDFT block nonetheless is prophesied at the second block. Matlab ${ }^{\circledR}$ models showed that the maximum peak power is nearly constant and nearly equal. Always in all cases the peak power can be prophesied in a manner similar to those in the next frontiers:

$$
\alpha \sigma_{2}^{2}<\max |x(k)|^{2} \leq \sigma_{2}^{2}
$$

Through Equation 2, it is possible to calculate the PAPR to produce

$$
\begin{aligned}
& P A P R=\frac{\alpha \sigma_{2}^{2}}{\sigma_{2}^{2}} \\
& \cong \\
& \cong 1.0[=0.0 \mathrm{~dB}]
\end{aligned}
$$

Therefore, the outcome for the M-PSK based OFDM signals is approximately $0.0 \mathrm{~dB}$.

Alternatively, the computational difficulty has been significantly decrease matched with that of the SLM method. Thus, the full amount of multiplication processes can be calculated by using this equation

$$
M_{2 D I D F T}=N \cdot \log _{2} N
$$

In place of the SLM method, the total number of multiplication operations is expressed as

$$
M_{S L M}=N \cdot U \cdot\left(1+0.5 \log _{2} N\right)
$$

The ratio decrease in the amount of multiplication processes can then be said by Equations 19 and 20, as follows:

$$
R_{M}=\left\{1-\frac{M_{2 D I D F T}}{M_{S L M}}\right\} \times 100 \%
$$

The amount of phase-rotation vectors is U used in the SLM method. Then, in this method the amount of addition processes is

$$
A_{2 D I D F T}=2 N \cdot \log _{2} N
$$

In place of the SLM method, the number of addition operations can be obtained from this equation:

$$
A_{S L M}=U \cdot N \cdot \log _{2} N
$$

Therefore, the ratio decrease is

$R_{A}=\left\{1-\frac{A_{2 D I D F T}}{A_{S L M}}\right\} \times 100 \%$

On behalf of sample, suppose that $\mathrm{U}=8$ and $\mathrm{N}=256$ subcarriers. At that time, M2DIDFT $=2048$, MSLM $=10240$, therefore that

$R_{M}=\left\{1-\frac{2048}{10240}\right\} \times 100 \%=80 \%$
Therefore, the decrease in the amount of multiplication processes for $\mathrm{U}=8$ alone is $80 \%$. As shown in the same input directly above, the amounts of addition processes for the suggested way and the SLM method are 4096 and 16,384, respectively. Finally, the percentage reduction in the addition operations is

$$
R_{A}=\left\{1-\frac{4096}{16384}\right\} \times 100 \%=75 \%
$$

The $75 \%$ decrease in the amount of addition processes accomplished in the study is one of the proposed scheme's advantages over the SLM scheme. An additional the amount of bits to the side data. The SLM technique requires four of side information bits, whereas our method requires none.

\section{RESULTS AND DISCUSSIONS}

Different scenarios are simulated in this section; using the entries shown in Table 1. For all charting commands of the PSK the PAPR values have already been predicted at $0.0 \mathrm{~dB}$

Table 1. model factors for three scenarios

\begin{tabular}{|c|c|c|c|}
\hline \# OFDM & $\mathbf{N}$ & $\mathbf{L}$ & $\mathbf{M}$ \\
\hline $100 \times 103$ & 64 & 4 & $4,8,32$, and 128 \\
\hline $16 \times 103$ & 128 & 4 & $4,8,32$, and 128 \\
\hline $16 \times 103$ & 256 & 4 & $4,8,32$, and 128 \\
\hline
\end{tabular}

\section{First scenario:}

Figure 3a (shown after third scenario) shows only the original OFDM PAPR values. This result is unlike that of the submitted structure, in which PAPR $\approx 0.0 \mathrm{~dB}$ for all Phases of the charting commands $(M=4,8,32$, and 128). Thus, Figure 3a shows 60 randomly selected PAPR values for both systems [original (1D-IDFT) and the suggested one (2D-IDFT)]. The PAPR values of the 2D-IDFT based system are all $0 \mathrm{~dB}$, whereas that of the original system reaches $14 \mathrm{~dB}$. Figure $3 \mathrm{~b}$ (shown after Figure 3a) shows results similar to those of Figure $3 \mathrm{a}$ but were obtained using the CCDF function. The results of the proposed system are not recognizable because all are approximately constant and equal to $0.0 \mathrm{~dB}$.

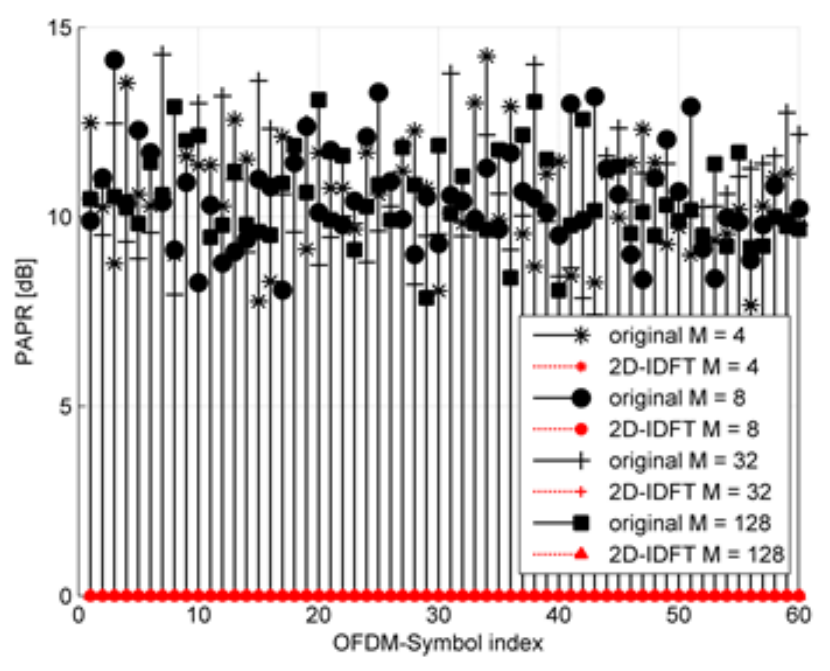

Fig 3a: PAPR values in dB for random 60 OFDM symbols of scenario $1(N=64)$. 


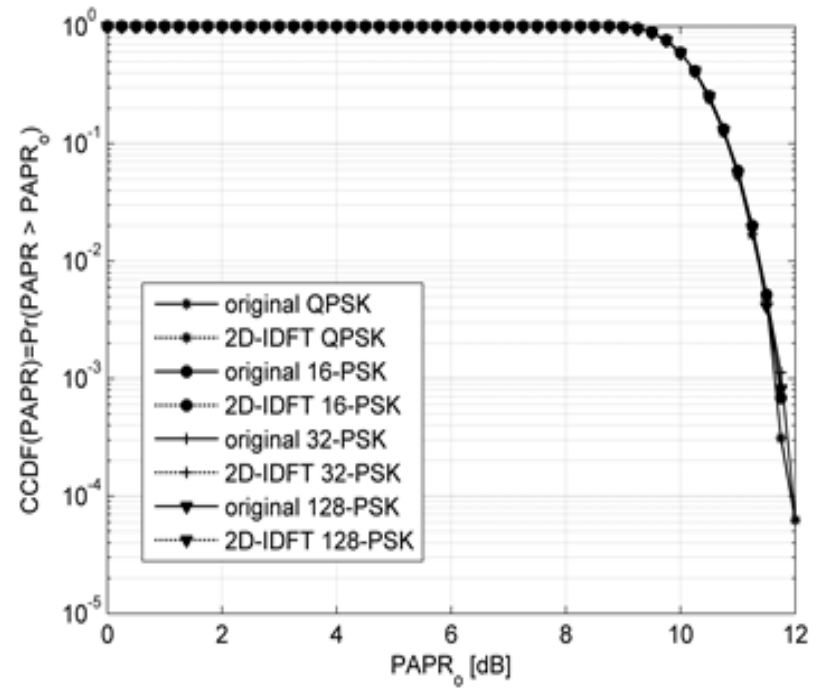

Fig 3b: CCDF results for scenario $1(N=64)$.

\section{Second scenario:}

On behalf of the next scenario, wherever $\mathrm{N}=128$ subcarriers, the estimated outcomes have been achieved. The stem plot is used to illustrate the PAPR values (Figure 4a (located after Figure 3b)), similar to that used in Figure 3a. Only 60 randomly selected OFDM symbols are displayed in the figure. The PAPR values of the suggested arrangement considerably reduced to nearly $0.0 \mathrm{~dB}$. Annotation that the extreme PAPR in Figure $4 \mathrm{a}$ be $16 \mathrm{~dB}$, while that in Figure $3 \mathrm{a}$ be $14 \mathrm{db}$. This case is common as when the amount of subcarriers upsurges, PAPR also increases. The conventional method used to measure the PAPR utilises the CCDF of the PAPR function (Figure 4b (shown after Figure 4a)). The recognisable results in the last figure are for the conventional system; the values for the 2D-IDFT based system cannot be detected. This nondetection is due to the nearly constant $(0.0 \mathrm{~dB})$ value, which is the goal of our proposed system.

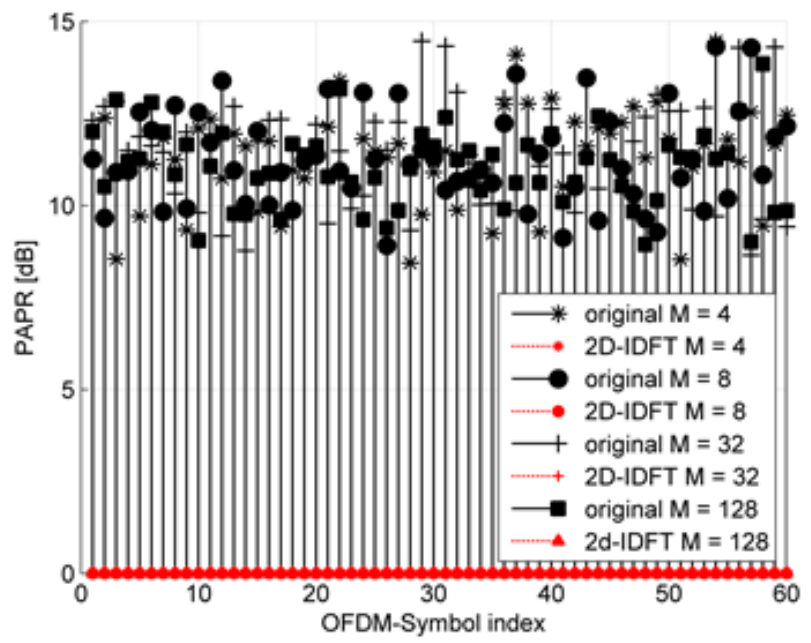

Fig 4a: PAPR values in dB for random 60 OFDM symbols of scenario $2(\mathrm{~N}=128)$.

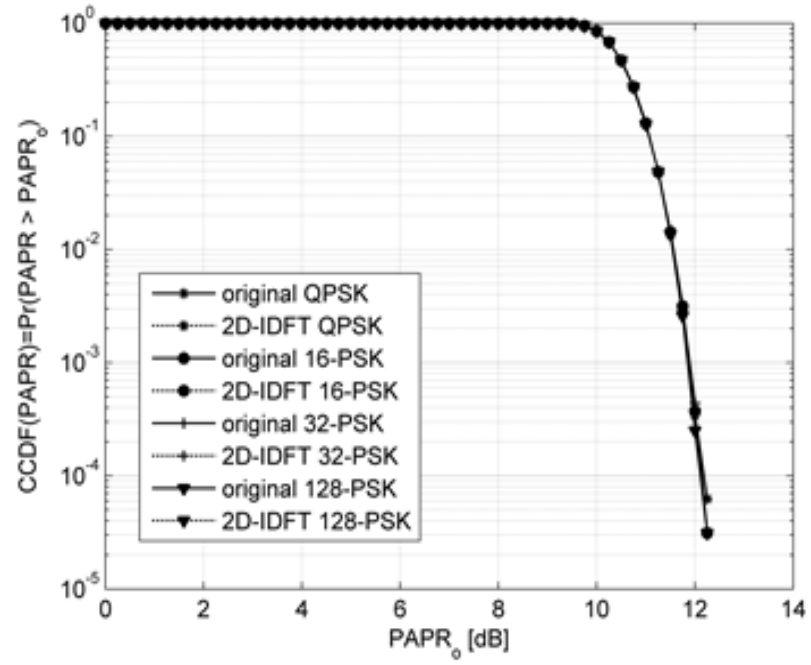

Fig 4b: CCDF results for scenario $2(\mathrm{~N}=128)$.

\section{Third scenario:}

Figure 5a (shown after Figure 4b) shows an illustration of the last scenario, in which $\mathrm{N}=256$ subcarriers. The plot type used in this figure is the same as those of the previous ones. As previously estimated, the PAPR for all M-PSK signals in Table 1 is reduced to $0.0 \mathrm{~dB}$. In addition, the maximum PAPR of the original system exceeds $16 \mathrm{~dB}$ because the increase in the number of subcarriers is greater than those of the others. The same predicted PAPR values are seen in Figure $5 \mathrm{~b}$ (located after Figure 5a) when the CCDF-PAPR function is used. These results are similar to those of the previous scenarios (Figures $3 b$ and $4 b$ ).

On the other hand, the BER of the suggested system exhibits no degradation (Figure 6 (shown after Figure 5a)) on behalf of state numeral one in Table top 1. This result indicates that the BER of the suggested system for 128 mapping levels, $\mathrm{N}=64$ subcarriers and $\mathrm{M}=4,8,32$ do not change or be affected in comparison with the original system. The same conclusions are obtained for scenarios 2 and 3 (Figures 7 and 8 (located after Figure 6)), respectively.

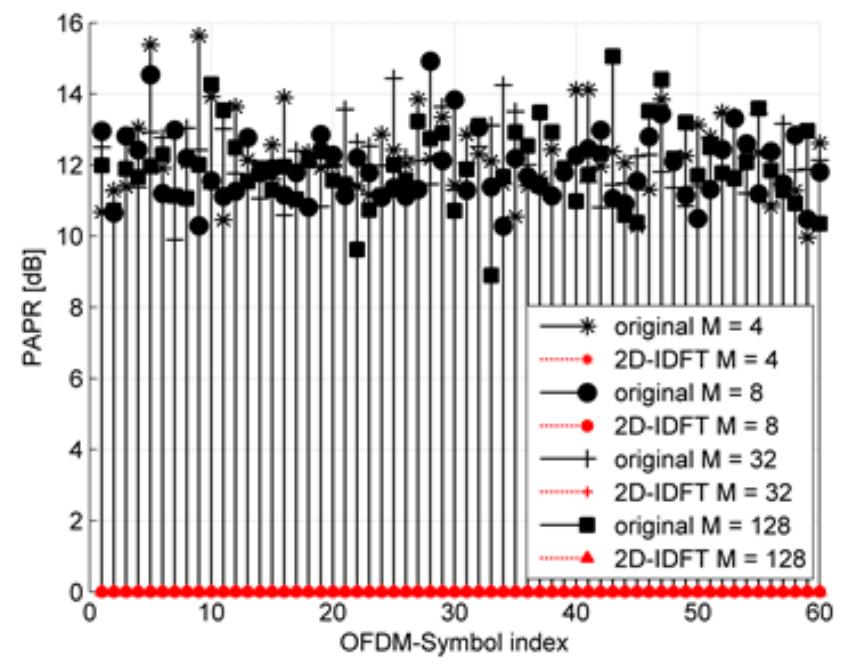

Fig 5a: PAPR values in $\mathrm{dB}$ for random 60 OFDM symbols of scenario $3(N=256)$. 


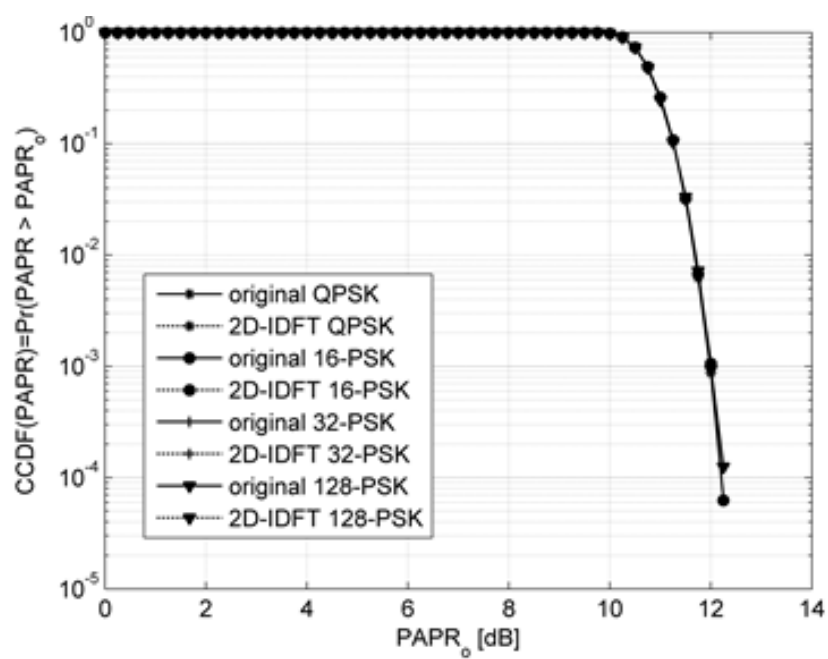

Fig 5b: CCDF outcomes for state three $(\mathrm{N}=\mathbf{2 5 6})$.

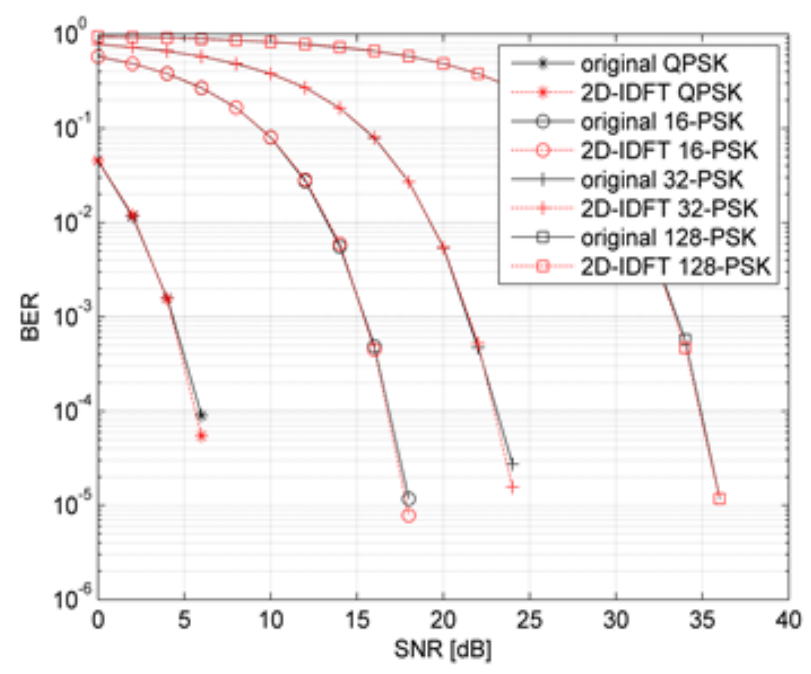

Fig 6: BER outcomes for state one $(N=64, M=4,8,32$, and 128)

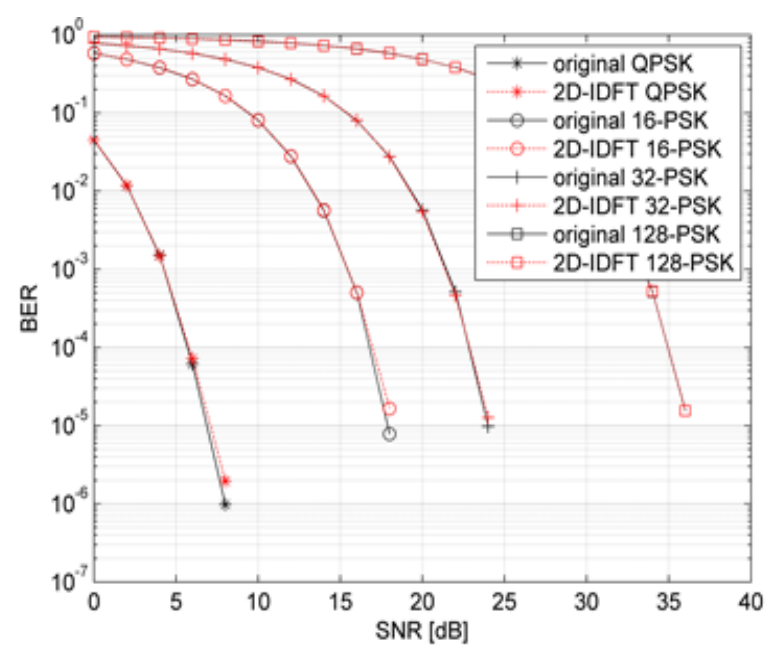

Fig 7: BER outcomes for state tow $(, M=4,8,32,128$ and $N$ = 128).

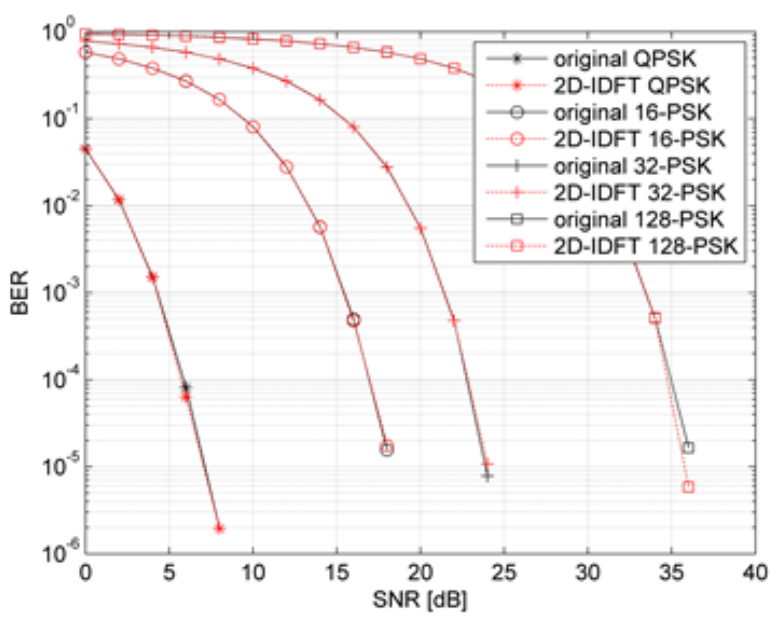

Fig 8: BER outcomes for state three $(M=4,8,32,128 \& N$ = 256).

\section{CONCLUSIONS}

In the OFDM system, the main problem is PAPR which is considered as the main weakness. To statement this problem a number of methods were established whichever at the expense of uncomplicatedness (i.e., the complication is increased by the necessity for side information, such as those in SLM, PTS) otherwise to calculate the performance of the BER. Consequently, In this work to reduce the deterioration in the performance of the BER, it was suggested using the 2D-IDFT Instead of using 1D-IDFT, to reduce the complexity of calculations and to neglect any additional side information. in this paper, a constant power envelope was completed for MPSK based OFDM signals.

\section{REFERENCES}

[1] Ahmed, M.S., Boussakta, S., Sharif, B.S. and Tsimenidis, C.C. (2011), 'OFDM Based on Low Complexity Transform to Increase Multipath Resilience and Reduce PAPR', Signal Processing, IEEE Transactions on, 59, 5994-6007. .

[2] Bauml, R.W., Fischer, R.F.H. and Huber, J.B. (1996), 'Reducing the peak-to-average power ratio of multicarrier modulation by selected mapping', Electronics Letters, 32, 2056-2057.

[3] Byoung-Jo, C., Ee-Lin, K. and Hanzo, L. (1999), 'Crestfactor study of MC-CDMA and OFDM', in Vehicular Technology Conference, 1999. VTC 1999 - Fall. IEEE VTS 50th, pp. 233-237 vol.231.

[4] Dae-Woon, L., Seok-Joong, H., Jong-Seon, N. and Habong, C. (2006), 'On the phase sequence set of SLM OFDM scheme for a crest factor reduction', Signal Processing, IEEE Transactions on, 54, 1931-1935.

[5] Di, W. and Spasojevic, P. (2008), 'Complementary Set Matrices Satisfying a Column Correlation Constraint', Information Theory, IEEE Transactions on, 54, 33303339 .

[6] Ermolova, N.Y. and Vainikainen, P. (2003), 'On the relationship between peak factor of a multicarrier signal and aperiodic autocorrelation of the generating sequence', Communications Letters, IEEE, 7, 107-108. 
[7] Freiman, G., Litsyn, S. and Yudin, A. (2004), 'A method to suppress high peaks in BPSK-modulated OFDM signal', Communications, IEEE Transactions on, 52, 1440-1443.

[8] Hussain, I.M. and Tasadduq, I.A. (2008), 'PAPR Analysis in OFDM Signals Based on Power Variance', in Wireless Communications, Networking and Mobile Computing, 2008. WiCOM '08. 4th International Conference on, pp. 1-4.

[9] Jae-Kwon, L., Ju-Hyun, Y. and Jin-Up, K. (2008), 'Estimation technique of low crest factor signal in multiple signal representation', in Advanced Communication Technology, 2008. ICACT 2008. 10th International Conference on, pp. 832-834.

[10] Ju, S.M. and Leung, S.H. (2003), 'Clipping on COFDM with phase on demand', Communications Letters, IEEE, 7, 49-51.

[11] Litsyn, S. and Wunder, G. (2006), 'Generalized bounds on the crest-factor distribution of OFDM signals with applications to code design', Information Theory, IEEE Transactions on, 52, 992-1006.

[12] Litsyn, S. and Yudin, A. (2005), 'Discrete and continuous maxima in multicarrier communication', Information Theory, IEEE Transactions on, 51, 919-928.

[13] Muller, S.H. and Huber, J.B. (1997), 'OFDM with reduced peak-to-average power ratio by optimum combination of partial transmit sequences', Electronics Letters, 33, 368-369.
[14] Paterson, K.G. and Tarokh, V. (2000), 'On the existence and construction of good codes with low peak-to-average power ratios', Information Theory, IEEE Transactions on, 46, 1974-1987.

[15] Proakis, J.G. and Salehi, M. (2008), Digital Communications, 5th Edition: McGraw-Hill Science/Engineering/Math.

[16] Taher, M.A., Mandeep, J.S., Ismail, M. and Mahdi, H.F. (2011), 'Blind technique to lower the PAPR of the MCCDMA system without complexity', in Communications (APCC), 2011 17th Asia-Pacific Conference on, pp. 688691.

[17] Tao, J. and Yiyan, W. (2008), 'An Overview: Peak-toAverage Power Ratio Reduction Techniques for OFDM Signals', Broadcasting, IEEE Transactions on, 54, 257268.

[18] Tellambura, C. (1997), 'Upper bound on peak factor of N-multiple carriers', Electronics Letters, 33, 1608-1609.

[19] Tellambura, C. (2001), 'Computation of the continuoustime PAR of an OFDM signal with BPSK subcarriers', Communications Letters, IEEE, 5, 185-187.

[20] Vallavaraj, A., Stewart, B.G. and Harrison, D.K. (2010), 'An evaluation of modified u-Law companding to reduce the PAPR of OFDM systems', AEU - International Journal of Electronics and Communications, 64, 844857.

[21] Zhu, X., Zhu, G. and Jiang, T. (2009), 'Reducing the peak-to-average power ratio using unitary matrix transformation', Communications, IET, 3, 161-171. 